\title{
INTERÉS PRIVADO Y PRODUCCIÓN ACTUAL DE CONOCIMIENTO EN CIENCIA, TECNOLOGÍA E INNOVACIÓN
}

\section{Héctor Eduardo Cardona Carmona ${ }^{1}$}

\section{Resumen}

Es un hecho que durante los últimos años vienen ocurriendo notorias transformaciones en la producción y apropiación del conocimiento en el contexto mundial. Partiendo de ello, en el presente artículo se defiende la tesis acerca de que la financiación, producción y apropiación del conocimiento científico y tecnológico en la sociedad contemporánea, se han convertido en prácticas que presentan una creciente tendencia a la privatización. Esta tendencia ha introducido una nueva concepción, en la que predominan intereses orientados a la mercantilización, comercialización y competitividad del conocimiento.

\section{Abstract}

It is a fact that during the last few years, remarkable changes in the production and the appropriation of the knowledge in the global context have been occurring. Based on this fact, this article intends to defend the thesis on that the financing, production and appropriation of the scientific

\footnotetext{
${ }^{1}$ Sociólogo - Magister en Educación y Desarrollo Humano. Estudiante del doctorado en Estudios sobre Ciencia y Tecnología y Gestión de la Innovación (Universidad del País Vasco). Investigador y docente de CTS (Ciencia, Tecnología y Sociedad) en el INSTITUTO TECNOLÓGICo METROPOLITANO. Contacto: hectorcardona@itm.edu.co.
}

and technological knowledge in the contemporaneous society have become practices that show an increasing tendency of the privatization. This tendency has introduced a new conception, in which interests oriented to the merchandising, commercialization and competitiveness of the knowledge predominate.

Palabras clave: Ciencia; Tecnología; Innovación; Financiación, producción y apropiación del conocimiento científico y tecnológico; Prácticas; Privatización; Intereses; Mercantilización, comercialización y competitividad del conocimiento.

Key words: Science, Technology, Innovation, Financing, production and appropriation of the scientific and technological knowledge, Practices, Privatization, Interests, Merchandising, commercialization and competitiveness of the knowledge.

\section{Introducción}

En el presente artículo se sostiene la tesis acerca de que la financiación, producción y apropiación del conocimiento científico y tecnológico en la sociedad contemporánea, presentan una tendencia a la privatización. Esta tendencia ha introducido una nueva orientación en el quehacer 
científico-tecnológico, en la que predominan intereses orientados a la mercantilización, comercialización y competitividad del conocimiento. Como sostiene León Olivé "el conocimiento se crea, se acumula, se difunde, se distribuye y se aprovecha. Pero ahora ya no todo el conocimiento es susceptible de apropiación pública, como había sido tradicionalmente, sino que una buena parte de él se compra y se vende entre particulares. Precisamente, parte de la novedad en la sociedad del conocimiento es que se han creado mercados del conocimiento. La apropiación privada del conocimiento, particularmente del conocimiento científico, es algo inédito en la historia" (Olivé, 2005: 50).

Teniendo presente que durante los últimos años vienen ocurriendo notorias transformaciones en la producción y apropiación del conocimiento en el contexto mundial, amerita plantear algunos interrogantes, como punto de partida para el análisis de la tesis antes enunciada:

¿Cómo se financia la investigación en la sociedad actual? ¿Hasta qué punto, puede afirmarse que existe una tendencia a la financiación y apropiación privada del conocimiento en el mundo actual? ¿Puede decirse que dicha tendencia se cumple de igual manera en la investigación científica, en la investigación tecnológicay en la investigación tecno-científica? ¿Qué rasgos caracterizan al conocimiento entendido como un bien privado? ¿Qué implicaciones tiene para los agentes dedicados a la producción de conocimientos, la tendencia a la financiación privada de la investigación? ¿Qué efectos genera el predominio de capitales privados dedicados a financiar la investigación tecno-científica, en el aprovechamiento de los resultados de la investigación? ¿Cómo se conjuga lo público y lo privado en las actuales formas de producción y apropiación de conocimiento?

En el primer apartado del artículo se analiza el problema de la naturaleza pública y privada del conocimiento producido en ciencia, tecnología e innovación, enfatizando en las posturas de algunos autores que defienden la naturaleza pública de dichos conocimientos. En el apartado dos, se presentan algunos datos y argumentos que contradicen la naturaleza pública en la actual producción de conocimiento y evidencian el predominio de la naturaleza privada. El apartado tres está dedicado a mostrar cómo la ciencia postacadémica, y la economía del conocimiento son correlatos de la privatización del conocimiento que han erosionado el ethos científico mertoniano. En la sección cuatro, se analiza las implicaciones éticas y políticas derivadas de la financiación y apropiación privada de la investigación tecno-científica. En el apartado cinco se desarrollan algunas conclusiones sobre el tema.

\section{Ciencia, tecnología e innovación: ¿conocimientos públicos o privados?}

Afirmar que existe una supuesta tendencia a la privatización del conocimiento en el mundo contemporáneo, no significa reconocer que todas las investigaciones e innovaciones de carácter científico y tecnológico producidas en diferentes ámbitos del conocimiento sean financiadas y apropiadas por el sector privado. Una cosa es afirmar que existe una tendencia a la privatización de la ciencia, la tecnología y la innovación y otra muy diferente es decir que todo el conocimiento científico y tecnológico que hoy se genera, procede de entidades privadas entre las que se encuentran universidades, empresas, industrias, laboratorios y centros de investigación. Para no caer en posturas reduccionistas, digamos que pese al predominio actual de la financiación y apropiación privada de la investigación científica y tecnológica, todavía existe una porción considerable de investigación financiada y apropiada públicamente.

Durante la década actual se han generado amplios debates acerca de la naturaleza pública y/o privada de la investigación ${ }^{2}$. Vega Encabo (2001) plantea que el uso 
del término ciencia pública no es unívoco ni uniforme en la literatura. Dos sentidos de público son esenciales para la controversia: el contraste público-secreto ${ }^{3}$ y la oposición público-privado en términos económicos (2001: 248). La oposición público-privado referida a la producción de conocimiento científico ${ }^{4}$, la define en los siguientes términos:

La ciencia es un bien público en cuanto opuesto a bien privado. Los resultados de la investigación científica pueden ser tratados como bienes económicos. En este sentido, la ciencia es un bien público porque sus resultados son no-apropiables y no-rivales ${ }^{5}$; por un lado, no se puede impedir que alguien que no ha contribuido a su producción se apropie de los resultados para su propio beneficio; por otro lado, no puede haber rivalidad entre dos personas para el uso de esos resultados. La cuestión es si existe algo intrínseco en los productos de la ciencia que haga de los mismos bienes públicos en sentido económico. Podría, en cambio, depender de otros factores, como del hecho de que esos resultados contribuyan al mantenimiento y provisión de otros bienes públicos no excluibles e indivisibles como el bienestar social, la educación, la sanidad, etc. (Vega

Encabo, 2001: 248).

En la misma línea de pensamiento que se acaba de citar, Broncano, apoyado en una de las más importantes teorías acerca de la naturaleza de las estructuras sociales, la teoría de los bienes públicos ${ }^{6}$, considera que no hay razones para

\footnotetext{
${ }^{2}$ En otras palabras, la investigación financiada con fondos públicos en la universidad y otros centros públicos de investigación y la investigación realizada con fondos privados en las empresas privadas esencialmente. Esta diferenciación es conveniente realizarla a la hora de analizar quién ha de ser el motor de la investigación y por qué. Una empresa privada por definición tiene como objetivo obtener beneficios a cambio de vender un producto 0 un servicio y satisfacer las necesidades de sus clientes en un determinado ámbito. Por esta razón la investigación en la empresa privada tiene como objetivo desarrollar nuevos productos o aumentar el valor añadido de los mismos.

${ }^{3}$ Afirma el autor que: "la ciencia es pública en cuanto opuesta a secreta. En algún sentido, este contraste está bien establecido. Las prácticas comunicativas institucionalizadas en los comienzos de la ciencia moderna tienden a liberar a los resultados científicos de su secreto. $\mathrm{E}$, independientemente de la influencia efectiva que se adjudique al sistema mertoniano de normas en las motivaciones reales de los científicos, hay algo en las prácticas de la ciencia que no premia mantener en secreto los resultados científicos. Ni siquiera la apelación a una supuesta ciencia militar que se mantiene en secreto (generalmente, por cuestiones de defensa nacional, aunque en ocasiones simplemente por interés tecnológico y estratégico) y alejada de la esfera del conocimiento público puede servir para poner en
}

considerar que el conocimiento científico actual pueda ser considerado como un bien privado. A este respecto afirma
Cabe argumentar, de modo pragmático y consecuencialista, que los costos de convertir la ciencia actual en un bien de club son altos y arriesgados, y quizá lo más importante, que nadie tiene la capacidad suficiente para planificar este cambio. Es cierto que algunas zonas de la investigación científica y tecnológica, como podría ser el caso de las biotecnologías, presentan aparentemente este sesgo, pero no creo que sea posible generalizar más allá de ciertos episodios locales. De hecho, para convertir la ciencia en un sistema de bienes de club habría quizá que convertir también el sistema educativo en un sistema de accesos limitados al conocimiento para prevenir un uso no respetuoso de los derechos privados de apropiación del conocimiento. Pero me parece que la razón de fondo para que los costos sociales de la privatización del conocimiento, y no sólo los económicos, sean extremadamente altos o directamente insoportables está en la naturaleza informacional del conocimiento: para impedir el uso de un cierto producto hay que impedir las condiciones cognitivas de acceso al producto y, por tanto, controlar el acceso al trasfondo necesario para su comprensión y posterior uso. Pero una reforma de este calibre del sistema educativo, convirtiéndolo en un sistema de puertas cerradas, pagaría un costo tan alto en términos de utilidad social del conocimiento que los beneficios locales estarían sobrepasados largamente por los costos globales. (Broncano, 2001: 26).

duda la viabilidad de la distinción. En realidad, la supuesta «ciencia militar» es parásita de las formas públicas de producción de conocimiento, y su extensión 0 generalización no sería más que un modo de estrangular las capacidades reales de avance científico. La presencia de reductos de secretismo en las actividades de la ciencia no afecta a las prácticas que sostienen la efectividad de la ciencia como sistema acumulativo de conocimientos. Su secreto no está en interés del avance de la ciencia sino de otro tipo de prioridades políticas. (Vega Encabo, 2001: 248).

${ }^{4}$ El autor hace referencia de manera exclusiva a la investigación científica, considera que en cuanto a la frontera entre lo público y lo privado, la gestión y producción de conocimiento tecnológico (sistema tecnológico) se rige con otras lógicas. "La ciencia tiene una naturaleza esencialmente pública, que no procede necesariamente de las características intrínsecas del conocimiento como bien público (económico) sino de la base de legitimación institucional de la producción de conocimiento científico. Este hecho permite mantener normativamente una distinción entre las actividades de la ciencia y de la tecnología en relación a los sistemas de concesión de derechos de propiedad sobre los resultados" (Vega Encabo, 2001: 247)

5 "Dos propiedades hacen de los bienes económicos bienes públicos: la no-rivalidad y la no-exclusividad. La primera podría caracterizarse en los siguientes términos: 


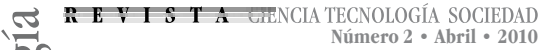 \\ 2. Tendencia a la privatización de la investigación tecno-científica}

Según los argumentos anteriores, no cabe concebir que la ciencia como práctica social contemporánea, pueda ser considerada un tipo de bien privado. No obstante, si prestamos atención a los datos que arrojan estudios realizados durante los últimos años acerca del estado actual de la investigación tecnocientífica en el mundo, los hechos ponen en tela de juicio el argumento acerca de la no viabilidad de la privatización de la ciencia. Afirmar que en los actuales momentos existe una tendencia a la privatización del conocimiento científico y tecnológico, implica, entre otros aspectos, examinar cuales agentes y agencias ${ }^{7}$ de la sociedad mundial, financian la investigación y se usufructúan de ella. Un argumento útil para dar respuesta a la pregunta por quién financia y produce la investigación en la sociedad contemporánea, es el que se desprende de las estadísticas acerca del número de investigadores que se dedican a la investigación financiada por capitales privados y los financiados por capitales públicos.

\section{Según la RICYT ${ }^{8}$, la cantidad de investigadores dedicados a la producción de conocimiento en el sector privado ha ido en aumento durante los últimos años.}

En América del Norte, durante la mayor parte de los años del período 1997- 2006, osciló entre el 70\% y el $80 \%$. Europa osciló entre el $40 \%$ y el $50 \%$, también con tendencia creciente, alcanzando prácticamente este valor en 2006. Los países de América Latina y el Caribe no fueron ajenos a este proceso de creciente importancia de las empresas (privadas) como sede de los investigadores y tecnólogos, sólo que su evolución transcurrió en niveles más bajos: entre 1997 y 1999 osciló entre el 10\% y el 20\%, con tendencia siempre creciente. Entre 2000 y 2004 osciló entre el $20 \%$ y el $30 \%$. Ya para 2005 el porcentaje superaba el 30\% y en 2006 se aproximaba al 40\%. (RICYT, 2008: 17).

Como puede verse en los datos anteriores, si bien es cierto que el comportamiento de la tendencia en el incremento de investigadores vinculados al sector privado es cuantitativamente diferente en los tres bloques de países presentados, esta diferencia no le resta validez a la tesis de que existe una tendencia al crecimiento de la producción de conocimiento en el sector privado y un declive en el sector público.

También Mitcham y Briggle, sostienen que aunque existan diferencias entre regiones en cuanto al porcentaje de fondos destinados a financiar la investigación privada, de todas maneras se evidencia una marcada tendencia al aumento de capitales privados, destinados a la I+D:

aplicación incrementa su valor; e) El conocimiento que produce no involucra competitividad en sus usos posteriores; f) Impredecibilidad de sus desarrollos futuros; g) Generadora de gastos de segundo orden, derivados de implicar a la colectividad, ya sea por acciones autoritarias, beneficios obtenidos del mercado o por la modificación de conductas morales. (Broncano, 2001: 24-25).

${ }^{7}$ Agente es la persona que actúa y provoca cambios y cuyos logros pueden juzgarse en función de sus propios valores y objetivos, independientemente de que los evaluemos o no también en función de algunos criterios externos [Sen, 2000: 35]. Para Fernando Broncano, la agencia es la disponibilidad que tienen los humanos para la transformación práctica, es decir, aquellas capacidades que tienen los humanos de intervenir en el mundo, entendiendo capacidades como aquellas disposiciones que no son actuales, sino futuras. En este sentido, la ciencia y la tecnología se constituyen en poderes, es decir, en capacidades humanas para controlar la realidad. Olivé comparte con Quintanilla la idea de que los agentes científicos y tecnológicos son colectivos humanos que actúan e interactúan para construir conocimiento. En este sentido, la ciencia y la tecnología son sistemas de acciones intencionales realizadas por agentes que tienen representaciones, intereses, normas, propósitos, fines, medios y resultados en común.

${ }^{8}$ Red Iberoamericana de Ciencia y Tecnología. 
Aunque la inversión en $\mathrm{I}+\mathrm{D}$ tienen niveles similares en las tres regiones principales de la OCDE, los tipos de inversiones y la estructura de las iniciativas de I $+D$ y los sistemas de innovación difieren de manera significativa. La ratio de inversión pública/privada varía de 1:5 en Japón a 2:3 en Francia. Así pues, el papel del sector privado en la financiación de la $\mathrm{I}+\mathrm{D}$ difiere marcadamente a lo largo de las tres principales regiones de la OCDE: supone casi tres cuartas partes de la I+D en Japón y el 63\% en los Estados Unidos, pero sólo un $55 \%$ en la Unión Europea. En los Estados Unidos, desde finales de la década de los 80 , el sector privado ha incrementado su inversión frente al sector público, y hay ahora una ratio de aproximadamente 2:1 entre los dos, aunque el sector privado tiende a centrarse en desarrollo más bien que en investigación [...] En muchos países asiáticos y en la Federación Rusa, el sector privado lleva a cabo la mayoría del desembolso en $\mathrm{I}+\mathrm{D}^{9}$. (Mitcham y

Briggle, 2007: 153- 154).

Con base en las estadísticas citadas en el texto anterior, es posible reconocer que la tendencia a la financiación de la investigación con fondos de carácter privado ha cobrado una mayor intensidad en países desarrollados. Como lo afirman Mitcham y Briggle, "en países no pertenecientes a la OCDE menos desarrollados, la mayoría del gasto en I $+D$ se lleva a cabo por el gobierno y sectores de educación superior" (Mitcham y Briggle, 2007:154). Pero aún así, en sociedades menos desarrolladas como es el caso de los

\footnotetext{
${ }^{9}$ La distinción que hacen los autores entre desarrollo e investigación forma parte de las definiciones para "Investigación y desarrollo" (I+D) de la Asociación Americana para el Avance de las Ciencias. Para la AAAS la investigación es "un estudio sistemático dirigido a un conocimiento científico más completo o a la comprensión del objeto de estudio". Esta puede ser básica o aplicada, dependiendo de si, cuando se busca el conocimiento, se tiene en mente una necesidad específica o no. Desarrollo se define como el "uso sistemático del conocimiento obtenido a partir de la investigación para la producción de materiales, aparatos, sistemas o métodos" (Mitcham y Briggle, 2007: 152). "El interés se fue desplazando desde la I+D en las décadas de la posguerra de mediados del siglo XX, hasta el actual énfasis en la innovación como motor generador de riqueza en el XXI. La importancia de la innovación tecnológica es reconocida en la teoría económica desde Marx en adelante, especialmente en la década de 1950. Pero desde finales de los ochenta del siglo XX, ha sido creciente la presencia de la innovación en los discursos políticos sobre ciencia y tecnología que antes sólo hablaban de I+D. A la I+D se añadió la i de innovación, y los fondos para ciencia y tecnología, antes separados entre los ministerios de educación (universidad y centros públicos de investigación) e industria (empresas y sector privado), comenzaron a repartirse tomando en cuenta los resultados (especialmente en términos de rendimientos comerciales) de las investigaciones realizadas con fondos públicos" (González de la Fe, 2009: 739).
}

países pertenecientes a América Latina, también se observa un aumento gradual de políticas que abogan por la incursión de capitales privados en la financiación de la investigación científica y tecnológica ${ }^{10}$.

¿Cómo entender esa aparente contradicción, entre los argumentos antes citados, que consideran poco probable la posibilidad de que la producción tecno-científica en los actuales momentos presente una tendencia a la privatización y los datos estadísticos que se acaban de presentar acerca de la situación actual de la ciencia en el mundo? La afirmación de Broncano en el sentido de que, "es cierto que algunas zonas de la investigación científica y tecnológica, como podría ser el caso de las biotecnologías, presentan aparentemente este sesgo, pero no creo que sea posible generalizar más allá de ciertos episodios locales" (Broncano: 2001, 26), es contradictoria si la contrastamos con los datos estadísticos presentados anteriormente.

\section{Ciencia postacadémica, Economía del Conocimiento y erosión del ethos mertoniano}

Aquí se comparte la tesis de Ziman, quien sostiene que el tipo de conocimiento denominado ciencia verdadera (real science), incluyendo la investigación que se desarrolla en las universidades, se está distanciando cada vez más del modo académico establecido por mucho tiempo. La investigación académica $^{11}$ está siendo complementada 0 invalidada

\footnotetext{
${ }^{10}$ En 1998, la media de la inversión de las empresas de ALC en I+D fue del $37,1 \%$ del total. Este valor ascendió al 41,5\% del total de los fondos invertidos en I+D por los países de la región en 2007. Este valor refleja fundamentalmente la situación de Brasil, cuyas empresas aportaron el 44,1\% de la inversión total del país, pero muestra también el avance que habría realizado Chile, que habría pasado de un 16,2\% en 1998 a un valor casi del doble en 2007 (29,3\%). Argentina declara también haber avanzado desde un valor del 27\% en 1998 hasta un 37,2\% en 2007. (Albornoz, 2009: 19).

${ }^{11}$ Se entiende aquí por ciencia o investigación académica el modelo de conocimiento que se construye en los espacios universitarios a través de la práctica de investigación orientada a la generación del conocimiento científico. A esta ciencia se le concibe como un conjunto de conocimientos objetivos y válidos. La objetividad de este conocimiento reside en el método científico que asegura la precisión y el rigor en la generación del conocimiento y la solidez de sus argumentos. Es una ciencia que, en su forma más extrema, algunos autores la consideran expresión del positivismo lógico, que da por sentado el poder del método científico y no concede atención seria a ninguna otra manifestación de la inteligencia humana, a ningún otro camino hacia la verdad o el entendimiento, a ninguna otra fuente de creación (Ziman, 1985: 63).
} 
por nuevas formas de producción del conocimiento denominada post-académica ${ }^{12}$. Los cambios en la forma en que se produce el conocimiento científico en la actualidad se deben tanto a factores externos como internos a esta actividad. Entre los externos, Ziman señala las presiones políticas, económicas e industriales que actúan cada vez con mayor fuerza sobre la comunidad científica.

Los internos, que son igualmente importantes que los anteriores, se deben a que la ciencia es un sistema dinámico y no una caja negra pasiva; tiene que adaptarse socialmente a las tensiones acumuladas que se generan al interior de la ciencia como resultado del rápido progreso científico y tecnológico (Ziman, 2000: 68).

Podemos definir el carácter novedoso de la ciencia postacadémica afirmando que en épocas anteriores a la actual, el conocimiento en general y los conocimientos científicos y tecnológicos en particular, siempre fueron considerados factores generadores de progreso y desarrollo de las sociedades, pero el auge que ha adquirido la valoración del conocimiento en la sociedad actual presenta unos rasgos

\footnotetext{
${ }^{12}$ La investigación post-académica se realiza normalmente por una sucesión de "proyectos". A medida que se intensifica la competencia por fondos, las propuestas de proyectos se vuelven más específicas en relación a los resultados esperados de la investigación, incluyendo su impacto social y económico más amplio. Esto ya no es algo que deben determinar los investigadores. Ya no se espera que las universidades y las instituciones de investigación se dediquen exclusivamente a la "búsqueda del conocimiento" en sí misma. Se ven impulsadas a buscar patrocinio industrial y a explotar al máximo cualquier descubrimiento patentable hecho por sus académicos, especialmente cuando se sospecha un posible beneficio comercial". (Ziman, 1999: 80-81)

${ }^{13}$ En este sentido, la novedad que caracteriza a la valoración del conocimiento en la sociedad actual, puede ser descrita en los siguientes términos:"Desde los clásicos de la economía y hasta hace poco tiempo, se sostenía que las fuentes del crecimiento económico y del bienestar de las naciones se encontraban en el stock o acervo de capital y la mano de obra disponible; mientras que elementos como el conocimiento científico y tecnológico, la educación y el capital humano, se consideraban exógenos a dichos procesos. Sin embargo, esta concepción ha evolucionado al punto que modernamente se reconoce que el crecimiento económico y la productividad se basan cada vez más en el conocimiento y la innovación, evolución que ha dado lugar a identificar otra etapa del capitalismo caracterizado con términos como "nueva economía" o "economía basada en el conocimiento" (the knowledge based economy). El conocimiento y su transformación en innovaciones, es decir invenciones (inventos) que han pasado la prueba del mercado, son los motores de la nueva economía" (Gallego, 2007: 151).
}

específicos que hacen de éste, el factor de generación de riqueza por antonomasia ${ }^{13}$.

Asociada al fenómeno de la privatización del conocimiento científico-tecnológico, durante las dos últimas décadas, en diferentes ámbitos tanto empresariales como académicos y en organismos encargados de orientar políticas públicas, ha cobrado fuerza la creencia de que la sociedad actual atraviesa por una nueva fase caracterizada por lo que se denomina la nueva economía o economía del conocimiento. La economía del conocimiento es una tendencia que ha introducido nuevas racionalidades reguladoras de todos los ámbitos y dinámicas de la vida social en el mundo contemporáneo.

Un argumento que es frecuente encontrar entre los defensores de la existencia de una nueva economía basadaen el conocimiento, sostiene que históricamente las sociedades humanas han tenido el conocimiento como un factor central de producción de riqueza asociada al desarrollo, pero que en la sociedad actual esa economía basada en el conocimiento cuenta con unas características que marcan rupturas con respecto a las dinámicas existentes en las sociedades anteriores. La producción de conocimiento en la economía del conocimiento se caracteriza por una estrecha interacción entre los modos científico, tecnológico e industrial de producción de conocimiento. La motivación de los agentes dedicados a la producción de conocimiento en dicho modelo es la comercialización y privatización de los productos del conocimiento, lo que ha generado que la valoración del conocimiento implique elevados niveles de competitividad ${ }^{14}$ para acceder a los recursos económicos necesarios para desarrollar su trabajo.

\footnotetext{
14 "Hoy la ciencia no puede subsistir sin grandes subvenciones estatales o privadas y éstas han dejado de crecer. Como consecuencia de todo ello, ha aumentado la competencia por los recursos, el culto por la eficiencia, la especialización y la búsqueda de resultados capaces de amortizar en breve plazo las enormes inversiones. Los grandes equipos de investigación propios de la Big Science están ahora también al servicio de compañías multinacionales con derechos de propiedad sobre los resultados de sus trabajos. La ansiedad por acaparar los derechos de patente y la agresividad de las campañas de marketing llega a afectar incluso a la investigación realizada en las universidades" (Diéguez, 2006: 177205).
} 
El mercado del conocimiento instaurado por la economía del conocimiento ha modificado también el tipo de conocimiento que es válido producir. Dentro de esta lógica se privilegia la búsqueda de investigación aplicada. Tal como lo afirma Gibbons:

El desarrollo de la ciencia ha alcanzado ahora una fase en la que muchos científicos han perdido interés por la búsqueda de los primeros principios. Están convencidos de que el mundo natural es una entidad demasiado compleja como para caer bajo una descripción unitaria que sea global y útil al mismo tiempo, en el sentido de que sea capaz de guiar la investigación posterior [...] El enorme aumento actual del interés por las aplicaciones sólo es parte de un reflejo de la persistencia de los intereses comerciales y militares en la ciencia y la tecnología. (Gibbons, 1997: 38).

La mercantilización del conocimiento, introduce nuevas formas organizativas para la producción y distribución del conocimiento. La producción de conocimiento forma parte de un proceso más amplio en el que el descubrimiento, la aplicación y el uso se hallan estrechamente integrados. Esto acontece por medio de un mecanismo que cobra cada vez mayor fuerza: la expansión del mercado para el conocimiento y la creciente comercialización de la ciencia y la tecnología ${ }^{15}$.

La valoración del conocimiento como "mercancía normal" convierte al conocimiento en algo que es valioso, siempre y cuando tenga un valor comercial; privilegiándose así su utilidad comercial y haciendo de él un bien privado. En esta lógica de funcionamiento, uno de los requisitos que deben tener las personas que se dedican a la investigación es la capacidad de acceder a fondos económicos que le garanticen permanecer en el sistema. De esta manera, el conocimiento

${ }^{15}$ Así describe Gibbons, este fenómeno: "La fuerza impulsora que se encuentra por detrás de la acelerada oferta y demanda de conocimiento comercializable está en la intensificación de la competencia internacional en los negocios y en la industria. En muchos casos, la investigación realizada dentro de la propia empresa ya no es suficiente para satisfacer las exigencias competitivas. Para comercializar el conocimiento, las empresas tienen que buscar nuevos tipos de vínculos con las universidades, los laboratorios gubernamentales y también con otras empresas" (Gibbons, 1997: 67). se constituye en factor de competitividad (factor de ventaja competitiva $)^{16}$. El carácter de competitividad por los recursos necesarios para desarrollar proyectos de investigación ha modificado el rol del investigador, quien se ve sometido a combinar su actividad investigadora con otro tipo de prácticas orientadas a la adquisición de fondos que posibiliten su quehacer.

Las prácticas investigativas inherentes a la ciencia postacadémica 0 economía del conocimiento presentan características que introducen rupturas radicales con los valores establecidos por el ethos mertoniano para la ciencia tradicional. En la nueva producción de conocimiento, valores como el desinterés y el comunitarismo (comunismo) son remplazados por valores antagónicos a éstos. En la producción de conocimiento actual, se observa una fuerte tendencia al predominio de valores $\mathrm{e}$ intereses regidos por la comercialización, mercantilización y competitividad que estimulan prácticas orientadas hacia fines comerciales con un explícito énfasis en el interés y la búsqueda de rentabilidad económica individual.

Concluyamos este apartado, citando el siguiente comentario de Núñez (2009), apoyado en Licha (1997), muy ilustrativo por cierto, acerca de la erosión del ethos mertoniano que se ha producido en las actuales prácticas de producción de conocimiento:

El ethos científico predicado por Merton y sus seguidores se refiere más bien al comportamiento - seguramente idealizado - de la ciencia académica. En las últimas décadas la ciencia se ha visto crecientemente involucrada en objetivos económicos y militares, lo que ha tenido profundas implicaciones en la práctica científica y en el

\footnotetext{
16 "La habilidad para obtener fondos se convierte, en sí misma, en un indicador de éxito [...] Los investigadores más astutos, al tratar de equilibrar su necesidad de equipo y personal con la de trabajar dentro de una estructura paradigmática dada, construyen sus carreras alrededor de una amplia base de financiación para la investigación. [...] En contraste con ellos, los científicos que se niegan a adoptar un enfoque estratégico para sus carreras, se enfrentan con la perspectiva de quedarse atrás, en la medida en que los consejos de investigación, las fundaciones y hasta las universidades ajustan sus recursos a nuevos horizontes" (Gibbons, 1997: 37).
} 
comportamiento mismo de los científicos. Surge entonces la duda razonable de hasta qué punto es sostenible hoy la clásica caracterización del ethos académico. Licha (1997) considera que se están produciendo cambios sustanciales en la ciencia académica lo que se revela a través de al menos dos evidencias: La nueva retórica sobre el deber ser de la investigación académica y las nuevas políticas para la ciencia orientadas al logro de la llamada 'competitividad global'. Las universidades y otras instituciones académicas se han visto envueltas en el objetivo de la competitividad, asentada en el cambio técnico y éste en el conocimiento científico. Como resultado de ello se profundiza el proceso de capitalización del conocimiento que la globalización se encarga de acelerar. El conocimiento se vuelve propiedad privada a través de la patentación de resultados de investigación, actividades de mercadeo, licencias y copyright y la formulación de políticas científicas con orientación comercial. Se impone así un modelo de ciencia llevado a cabo por un científico cuyo comportamiento es muy semejante al de los empresarios, muy preocupado por captar fondos y generar ingresos y cuyo trabajo se valora cada vez más en términos económicos y empresariales. Es obvio que todo esto afecta las normas y valores clásicos y se altera la cultura institucional y la misión de la investigación académica. El desinterés, el comunitarismo ceden paso a la búsqueda de beneficios y status. (Núñez, 2009: 1).

\section{Implicaciones éticas y políticas derivadas de la financiación y apropiación privada de la investigación tecno-científica}

¿Qué implicaciones éticas y políticas tiene para los agentes dedicados a la producción de conocimientos y para el tipo de conocimiento que se produce, este comportamiento que se acaba de presentar relacionado con la tendencia creciente a la financiación privada de la investigación? La tendencia creciente a la financiación de la investigación tecno-científica con recursos de carácter privado, ha generado algunas críticas acerca de las implicaciones éticas y políticas que se derivan de este fenómeno.

Según Ziman (2003), una ciencia que no se limite a lo puramente instrumental debería ser: pública, universal, imaginativa, autocrítica y desinteresada. Pero, esta lista de valores se encuentra en clara oposición con el modo en que la ciencia desarrolla habitualmente las funciones que los poderes sociales le demandan en la actualidad. En efecto, la tecno-ciencia que se practica hoy suele producir sobre todo un conocimiento que es patentable, particular o local, prosaico, pragmático e interesado 0 parcial. Esta modalidad ha transformado profundamente la estructura de la práctica científica-tecnológica en todas sus dimensiones y también ha incorporado nuevos valores a la actividad científica, incidiendo así profundamente en su axiología (Echeverría, 2003).

De otro lado, Vega Encabo advierte que:

Una tendencia generalizada a la privatización de la ciencia (en términos económicos) podría acarrear consecuencias indeseables para el equilibrio institucional de los sistemas de producción de conocimiento y afectar a la legitimidad de la institución científica. La ciencia tiene una naturaleza esencialmente pública, que no procede necesariamente de las características intrínsecas del conocimiento como bien público (económico) sino de la base de legitimación institucional de la producción de conocimiento científico. Este hecho permite mantener normativamente una distinción entre las actividades de la ciencia y de la tecnología en relación a los sistemas de concesión de derechos de propiedad sobre los resultados, y revisar argumentos políticos sobre la financiación de la ciencia" (Vega Encabo, 2001: 247).

La crítica de Vega Encabo a la tendencia privatizadora del conocimiento científico, está orientada a develar los problemas de comunicación y aprovechamiento de los resultados de la investigación científica, como consecuencia de la tendencia a la mercantilización del conocimiento. La inversión pública y privada en la financiación de la investigación científica y tecnológica cuenta con intereses diferentes. Para decirlo escuetamente, la primera propende la construcción de conocimiento cuyos resultados tienen un carácter incluyente, democrático y participativo. La inversión privada en la investigación científica y tecnológica 
persigue intereses de ganancia económica y en este sentido es excluyente y sus resultados son inaccesibles para amplias capas de la sociedad. "El mecanismo del mercado tiene una tendencia a desalentar la producción de bienes públicos a causa de una incapacidad por parte de los productores para apropiarse completamente del valor de los frutos de sus esfuerzos" (Dasgupta y David, 1994: 496).

Otra de las críticas que se hace al auge inusitado del carácter privado (conocimiento como bien privado) en las prácticas científicas y tecnológicas está relacionada con el fenómeno de las patentes. Como sostiene González de la Fe,

\section{[...] las patentes son el símbolo científico por excelencia} de lo que se conoce como Modo 2 de hacer ciencia. Los centros y los laboratorios las persiguen como señal de éxito de sus proyectos y se les evalúa por el número y la rentabilidad de las mismas. Los saldos de la balanza tecnológica, de la dependencia tecnológica, tiene en las patentes y las licencias su principal indicador. La industria del conocimiento se desarrolla en torno a ellas y la competencia es una carrera por patentar primero y de forma más amplia (González de la Fe, 2004: 7).

También desde la perspectiva de estudios de las políticas de ciencia y tecnología ${ }^{17}$ surgen algunas críticas que consideran que las actuales políticas de ciencia y tecnología se reducen a ser políticas del presupuesto científico, dejando de lado el hecho de que las aportaciones a la investigación reviertan en beneficios sociales. A este respecto, plantean Mitcham y Biggle lo siguiente:

Aunque podemos tener dudas acerca de reducir la política científica a presupuestos, es instructivo adoptar esta perspectiva especialmente porque saca a relucir temas filosóficos. Proporciona una visión sinóptica de quién está financiando qué tipo de investigación a qué niveles. Puede sentar las bases de comparaciones sociológicas sobre cómo esas variables difieren en

${ }^{17}$ Conjunto de medidas que toma el gobierno de un Estado o unión de Estados con el fin de fomentar con dinero público " el desarrollo de la investigación científica, el proceso de la innovación tecnológica, o el uso de la ciencia y la tecnología para objetivos políticos generales" ( Moya, 1998: 91). distintos países. Y por último, plantea cuestiones éticas sobre la distribución de las inversiones en investigación y los beneficios resultantes. Un tema ético que la políica del presupuesto científico saca a relucir es el uso justo de los recursos limitados. Aunque el modelo lineal sugiere que no debemos preocuparnos por un exceso de investigación científica, los políticos se enfrentan a la realidad de tener que sacrificar unas cosas por otras. ¿Quién debería poner los fondos? ¿Para desarrollar qué tipo de investigación científica? ¿A quién debería beneficiar esto? ¿Qué tipo de ciencia es más importante financiar? (Mitcham y Briggle, 2007: 152).

Algunos de los debates éticos y políticos que presenta la investigación científica y tecnológica realizada según el "modelo de mercado"18, son a juicio de los autores que se acaban de citar, los siguientes: a) los gobiernos deberían concentrarse en la investigación básica, porque sus beneficios son especulativos y a largo plazo, haciéndola así menos atractiva para organizaciones que se mueven por la mera búsqueda de beneficios económicos; b) el incremento de conexiones entre empresas y universidades hace peligrar la libertad de la investigación, en tanto que se prioriza la regulación de la información y de los productos para usos rentables, frente al libre intercambio de ideas; y c) el "complejo academia-industria" suscita también cuestiones sobre si la búsqueda de beneficios económicos distorsionaría el bien público o si los conflictos de intereses impedirían informar de los resultados negativos.

\section{Conclusiones}

Es una postura reduccionista pretender afirmar que la actual producción y apropiación de conocimiento en el ámbito mundial, cualquiera que sea el nombre que le demos al conocimiento (científico, tecnológico, tecnocientífico, $\mathrm{I}+\mathrm{D}+\mathrm{i}$ ), son prácticas 0 actividades de dominio exclusivo de los intereses provenientes de capitales privados,

\footnotetext{
${ }^{18}$ El "modelo de mercado" es aquel en el que la dinámica de la demanda de los consumidores y los beneficios de las empresas dictan los tipos de investigación que se pueden realizar.
} 
y que la naturaleza pública del conocimiento es un asunto del pasado. No es posible generalizar la afirmación anterior, debido a varias razones que muestran las múltiples dimensiones de este fenómeno; entre ellas las siguientes: a) no existe un conocimiento científico o tecnológico que se pueda agrupar, en términos absolutos, en una sola categoría denominada conocimiento privado; b) existen diferencias en cuanto al financiamiento y apropiación entre la ciencia, la tecnología, la tecno-ciencia y la innovación; c) en el campo de las ciencias existen diferencias de comportamiento (no es lo mismo la financiación y apropiación de resultados en las ciencias básicas que en las ciencias aplicadas); d) existen algunos países y sistemas sociales de conocimiento que reivindican la importancia de la inversión y apropiación pública del conocimiento y otros países que privilegian la participación de capitales privados en la producción de ciencia, tecnología e innovación.

Como se mencionó en uno de los apartados anteriores, diferentes autores presentan argumentos que sostienen la imposibilidad de que la ciencia pueda constituirse en un bien privado, por una serie de características que hacen de ella un bien público.

A este respecto, es menester precisar, que si bien es cierto que no puede generalizarse el enunciado acerca de que toda la ciencia que actualmente se produce en el mundo presenta la tendencia a ser valorada como bien privado, también es cierto que las nuevas formas de producción de conocimiento científico-tecnológico (tal como se ha mostrado en las estadísticas que se han presentado) muestran una tendencia creciente a la financiación y apropiación por parte de intereses económicos de carácter privado.

Uno de los aspectos que se discutió en el artículo, es la incidencia que se puede observar de la economía del conocimiento y los hechos económicos vinculados con la aplicación económica del conocimiento. Dicha valoración del conocimiento ha modificado la concepción tradicional del ethos científico y ha introducido nuevos valores en las prácticas de ciencia, tecnología e innovación. A este respecto firma Olivé que

"[...] los productos de la tecno-ciencia resultan del trabajo de grupos de científicos, de tecnólogos, de administradores y gestores, de empresarios e inversionistas y muchas veces de militares. $Y$ aunque no es una característica intrínseca de la tecno-ciencia, hasta ahora el control de los sistemas tecno-científicos ha estado en pocas manos de élites políticas, de grupos dirigentes de empresas transnacionales o de militares, asesorados por expertos tecno-científicos. Este es un rasgo de la estructura de poder mundial en virtud del cual, además del hecho de que el conocimiento se ha convertido en una nueva forma de riqueza que puede reproducirse a sí misma, también es una forma novedosa de poder. Esto ha trastocado los sistemas de valores en la producción y circulación del conocimiento. Ahora valores económicos, como la ganancia, han pasado a formar parte de la estructura axiológica de muchos sistemas tecnológicos y tecnocientíficos (Olivé, 2007: 55).

\section{Bibliografía}

Albornoz, M. (2009). El Estado de la Ciencia. Principales Indicadores de Ciencia y Tecnología Iberoamericanos/Interamericanos. RICYT. Buenos Aires (Argentina).

Broncano, F. (2001). “¿Es la ciencia un bien público?”. Revista Claves de Razón Práctica, $\mathrm{N}^{\circ} 115$. 22-28.

Buch, T. (1999). Respuesta al artículo titulado "La Ciencia como ética" de John Ziman. El Cultural - 17.10.99. Suplemento dominical del diario El Mundo (Madrid). Páginas 80-81.

Dasgupta, P. y David, P. A. (1994). Toward a New Economics of Science. Research Policy, $\mathrm{N}^{\circ}$ 23. 487-521. 
Diéguez, A. (2006). La ciencia desde una perspectiva postmoderna: Entre la legitimidad política y la validez epistemológica. Universidad de Málaga. Actas de las II Jornadas de Filosofía: Filosofía y política. Coín, Málaga: Procure. 177-205.

Gallego Alzate, J. (2007). Economía del conocimiento e innovación. En: Ética, innovación y estética. Fondo Editorial Instituto Tecnológico Metropolitano. Medellín (Colombia).

Gibbons, M; Limoges C; Nowotny H; Schwartzman, S; Scott, P. y Trow M. (1997). La nueva producción del conocimiento. La dinámica de la ciencia y la investigación en las sociedades contemporáneas. Ediciones Pomares- Corredor, S. A, Barcelona.

González de la Fe, T. (2004). Desigualdad y propiedad privada en la sociedad del conocimiento. Memorias VII Congreso Español de Sociología. Alicante (España).

González de la Fe, T. (2009). El modelo de triple hélice de relaciones universidad, industria y gobierno: un análisis crítico". ARBOR Ciencia, Pensamiento $y$ Cultura CLXXXV No. 738. 739-755.

Mitcham, C. y Briggle A. (2007). Ciencia y Política: Perspectiva Histórica y Modelos Alternativos. Revista Iberoamericana de Ciencia, Tecnología y SociedadCTS, abril, año/vol. 3, número 008. Redes. Centro de Estudios sobre Ciencia, Desarrollo y Educación Superior. Buenos Aires, Argentina.

Moya, E. (1998). Crítica de la razón tecnocientífica. Madrid. Biblioteca Nueva.

Núñez Jover, J. (2009). La ciencia y la tecnología como procesos sociales. Lo que la educación científica no debería olvidar. Comunidades científicas, retos y paradigmas. Sala de Lectura Boletín CTS. Organización de Estados Iberoamericanos. http://www.oei.es/salactsi/ nunez06.htm. Recuperado 3 de junio de 2010.

Olivé, L. (2005). La cultura científica y tecnológica en el tránsito a la sociedad del conocimiento. Revista de la Educación Superior Vol. XXXIV (4), №. 136. ISSN: 01852760. ANUIES, México.

Olivé, L. (2007). La Ciencia y la Tecnología en la Sociedad del Conocimiento. Ética, política y epistemología. Fondo de Cultura Económica. México.

RICYT. (2008). El Estado de la Ciencia. Principales Indicadores de Ciencia y Tecnología Iberoamericanos / Interamericanos. Organización de Estados Iberoamericanos.

Sen, A. (2000) Desarrollo y Libertad. Barcelona: Editorial Planeta.

Vega Encabo, J. (2001). Ciencia privada, conocimiento público. Algunas determinantes de las controversias políticas en la era de la tecnociencia. Isegoría, 0(25). 247-261.

Ziman, J. (1999). La Ciencia como ética. El Cultural Suplemento dominical del diario El Mundo. Madrid. 80-81.

Ziman, J. (2000.) Real Science. What it is, and what it means, Cambridge University Press, Cambridge, Inglaterra.

Ziman, J. (2003). Ciencia y sociedad civil. Revista Iberoamericana de Ciencia, Tecnología y Sociedad, 1(1). Organización de Estados Iberoamericanos (OEI). 177-188.

Fecha de recepción: 8 de abril de 2010 Fecha de aceptación: 3 de mayo de 2010 


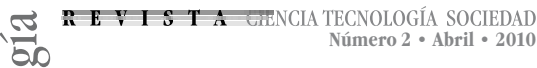

을 


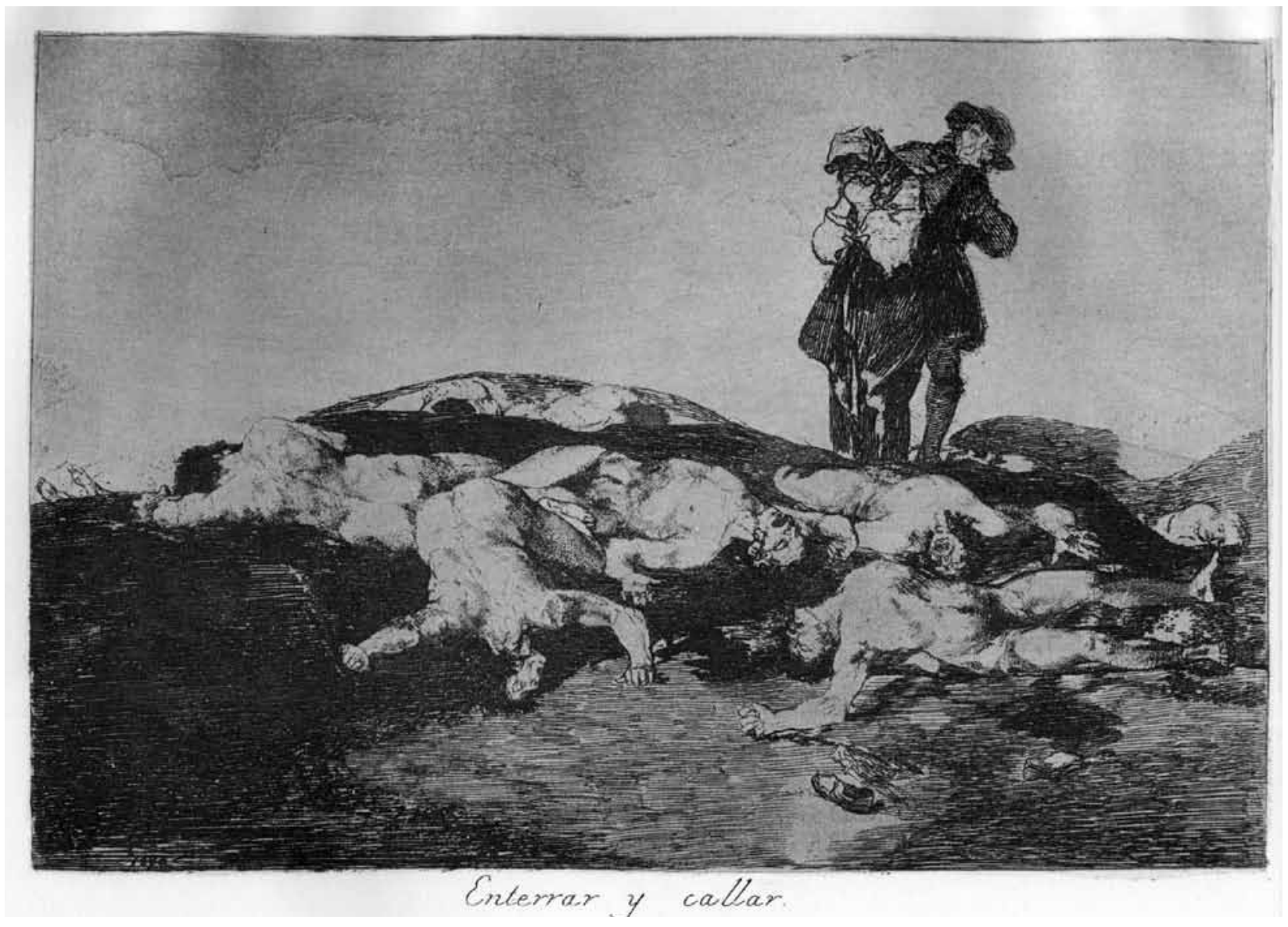

Título:

Enterrar

y callar 